\title{
Situación y desafíos en el campo de los recursos humanos en salud en el Área Andina, en los albores de los 2000
}

\author{
JUAN ARROYO \\ Facultad de Salud Pública y Administración, Universidad Peruana Cayetano Heredia.
}

\section{RESUMEN}

El artículo presenta una revisión de la situación actual y desafíos de los recursos humanos en salud en cinco países andinos: Bolivia, Colombia, Ecuador, Perú y Venezuela. Reseña transversalmente los problemas comunes al área andina, sobre la base de estudios por países. Se concluye que en los países andinos hay un tránsito muy inicial a la nueva situación de los recursos humanos, diferenciándose tres situaciones sobre recursos humanos: a) situación de flexibilización laboral avanzada y en medio de un cambio sistémico (Colombia); b) situación inversa, de países con una muy lenta asimilación de los cambios reformistas, persistencia del modelo previo y fuerte resistencia gremial a los cambios (Ecuador, Bolivia y Venezuela); y c) situación sui generis de fragmentación del régimen laboral, retroceso de los nombramientos e incremento de las contrataciones, ingreso con fuerza del modelo gerencial y presencia gremial médica importante con incidencia en el campo de recursos humanos (Perú). Se reseña ocho temas pendientes en el campo de los recursos humanos: 1) el paso de la baja disponibilidad a la sobreoferta urbana; 2) el desafío irresuelto del nuevo régimen laboral, en pleno tránsito de los nombramientos a las contrataciones; 3 ) el empobrecimiento de los salarios, el doble régimen salarial usando los proyectos de cooperación y la necesaria reinvención de la carrera pública; 4) el difícil balance entre costos y calidad, con el desplazamiento del arte clínico a la medicina gerenciada; 5) la paradójica disminución del mercado laboral y a la vez el incremento de la demanda educativa, poniendo en relieve la formación de calidad y la acreditación; 6) el paso de la formación clásica universitaria a la educación continua y permanente, y a la recertificación profesional; 7) la renovación pedagógica en salud, con los nuevos enfoques educativos; y 8) el tránsito en el campo de los recursos humanos de la débil regulación a la desregulación, con el consiguiente debilitamiento de la gobernabilidad de los procesos que afectan más directamente a los recursos humanos en salud.

Palabras claves: Recursos humanos en salud; salud pública; países en desarrollo. HEALTH HUMAN RESOURCES IN THE ANDEAN AREA: SITUATION AND CHALLENGES AT THE
BEGINNING OF THE 2000s

SUMMARY

The article presents a review of the current situation and challenges in the field of health human resources at five Andean countries: Bolivia, Colombia, Ecuador, Peru and Venezuela. The article makes a transversal review of common problems in the Andean area, based on studies per countries summarised in the initial chapter of the report, which has not been published here. The study was performed between August and November 2000, based on secondary sources and consultation with experts in the subject from the five Andean countries. The conclusion is that the Andean countries are arriving to a very initial transition stage to the new human resources situation defined by sector reforms postulates. We can distinguish three situations on human resources: a) a situation of advanced labour flexibility, within a systematic change (Colombia); b) an opposite situation, countries with a very slow reform changes assimilation, the previous model persists and the union resistance to the changes is very strong (Ecuador, Bolivia and Venezuela), and c) a sui generis situation: labour regime fragmentation, decreasing appointments and increasing contracts, the managerial model enters strongly and the presence of the medical union is important with influence in the human resources area (Peru). A description of eight pending matters is provided: 1) the passage from the low availability to the oversupply at urban level; 2) the unsolved challenge of the new labour regime, in transition from appointments to contracts; 3) the salary impoverishment, the double salary regime using cooperation projects and the required reinvention of the public career; 4) the hard balance between costs and quality with the displacement from the clinical art to the managerial medicine; 5) the paradoxical reduction of the labour market and the increasing educational demand, underlining qualified training and accreditation; 6) the transit from the classic university training to the continuous and permanent education and the professional re-certification; 7) the health area pedagogical renewal, including new educational approaches; and 8) the human resources area transit from weak regulation to deregulation, and consequent governability processes weakening that affects more directly to the health area human resources.

Key Words: Health menpower; public health; developing countries.

Correspondencia:

Dr. Juan Arroyo Laguna

Av. Honorio Delgado 430

Lima 31, Perú

E-mail:juanarroyo@terra.com.pe 
La revisión realizada de la situación de los recursos humanos en salud en este lado del continente muestra que en los países andinos hay un tránsito inconcluso y muy inicial a la nueva situación de los recursos humanos, definida por los postulados de las reformas sectoriales. Salvo Colombia, que es un caso de reforma global avanzada, todos los demás países andinos están en las primeras etapas de la reforma, que además no aparece bajo la forma de proyectos sectoriales de reforma, sino como un mosaico de reformas meso y micro.

Esto repercute en el campo de los recursos humanos, superponiendo viejos y nuevos problemas. En realidad, muchos suponen que la reforma ha estado toda la década luchando contra el modelo burocrático-estatista en la gestión pública, cuando en verdad, en nuestros países, el modelo burocrático nunca terminó realmente de imponerse sobre el más resistente de todos los modelos de administración pública en nuestras latitudes, el patrimonialismo o clientelismo. El desorden y la descoordinación no son fruto del burocratismo, que es ordenado y sistémico, sino de este estilo premoderno de gestión, que viene de antiguo. Burocratismo y patrimonialismo, aunque conflictivamente, siempre coexistieron y coexisten aún. Había por eso en la subregión países más "burocratizados" y otros más "tradicionalistas", países con "sistemas" de salud reales y otros en que el nombre de "sistemas de salud" no expresaba bien la realidad de servicios profundamente fragmentados. La nueva reforma en salud de los 90 se ha venido implantando sobre esta realidad, originándose un nuevo tránsito también inconcluso.

Nuestro modelo de gestión sectorial resultante ahora, luego de una década de reforma, es por eso una mixtura, sólo que una nueva mixtura, que superpone el patrimonialismo, el burocratismo y el gerencialismo. Se observa, por eso, a la vez la administración en base a confianzas, la cultura burocrática centrada en los procedimientos y las ideas-fuerza de la eficiencia y la administración en base a resultados.

Este esquema dinámico de varios modelos de gestión en continua pugna y articulación nos parece tiene más capacidad explicativa y potencia interventora en los países andinos que el esquema bipolar "situación nueva" vs. "situación anterior". Efectivamente, en un sentido la situación es nueva, pero por razones diferentes: hay en general una desestabilización del régimen laboral burocrático y del modelo burocrático-estatal, pero sin que éste sea reemplazado plenamente por el régimen flexible impulsado por las reformas de primera generación y sin que se instalen -salvo muy parcialmentemecanismos de pago basados en sistemas de incentivos, como preveen los modelos de reforma.

En otras palabras, en las condiciones particulares del área andina, la desestabilización y la desregulación no han generado espacio sólo o principalmente al mercado, sino más bien desinstitucionalizado lo poco avanzado en la regulación de los recursos humanos y otorgado espacio para el reavivamiento del modelo clientelar en las relaciones laborales. El retroceso del escalafón burocrático o su afectación parcial no ha dado lugar a sistemas meritocráticos, sino más bien expandido la discrecionalidad del contratista, lo que en países de baja institucionalidad no significa necesariamente promover la gerenciabilidad sino la toma de decisiones ventajista propia del régimen clientelar. De ahí la desaparición de los concursos, la expansión notoria de los cargos de confianza y la construcción de lealtades con los pagos por bonificaciones en algunos países.

La institucionalidad en salud no ha podido, por tanto, escapar de las condiciones propias de nuestros países andinos, sumamente heterogéneas. La ola de reformas anterior a la actual se basó en el modelo burocrático estatal. Construyó sistemas de salud, formó funcionariados nacionales, formuló normatividad legal, asalarió a los profesionales, introdujo la planificación normativa, en una palabra intentó dotar de racionalidad weberiana a lo público, si bien con su conocido flanco débil de sobredimensionar los procedimientos (Tabla 1).

Debió confrontar para eso al modelo patrimonial, que no distingue lo público de lo privado, en que se administra empíricamente y sobre la base de la más amplia discrecionalidad del decisor, casi sin normas legales, primando los criterios de clientelismo y confianza. Los recursos humanos de la etapa de las profesiones liberales ingresaron como empleados públicos a los nuevos estados 
Tabla 1.- Etapas y modelos de gestión en salud.

\begin{tabular}{|c|c|c|}
\hline Modelo patrimonialista & Modelo burocrático & Modelo gerencial \\
\hline \multicolumn{3}{|c|}{ En la gestión en general } \\
\hline $\begin{array}{l}\text { - No diferencia lo público } \\
\text { de lo privado } \\
\text { - Procedimientos rudimentarios } \\
\text { - Escaso profesionalismo } \\
\text { - Clientelaje y prebendalismo }\end{array}$ & $\begin{array}{l}\text { - Sistemas de salud } \\
\text { - Vértice técnico } \\
\text { - Gestión racional } \\
\text { - Rendimientos medios } \\
\text { - Identidades institucionales } \\
\text { - Institucionalización } \\
\text { - Énfasis en procedimientos, } \\
\text { medios y procesos }\end{array}$ & $\begin{array}{l}\text { - Énfasis en resultados e impacto } \\
\text { - Flexibilidad en los procedimientos } \\
\text { - Énfasis en evaluación del } \\
\text { desempeño } \\
\text { - Gerencia y planeamiento } \\
\text { estratégicos } \\
\text { - Gerencia de la calidad } \\
\text { - Gerencia de proyectos y programas }\end{array}$ \\
\hline \multicolumn{3}{|c|}{ En el campo de los recursos humanos en salud } \\
\hline $\begin{array}{l}\text { - Regulación muy escasa o nula } \\
\text { - Amplia discrecionalidad } \\
\text { del decisor } \\
\text { - Manejo laboral sobre la base } \\
\text { de confianzas y lealtades } \\
\text { - Debilidad técnica } \\
\text { - Fragmentación y feudalización } \\
\text { de las instituciones y cargos } \\
\text { - Primacía de la casuística }\end{array}$ & $\begin{array}{l}\text { - Régimen legal } \\
\text { - Estabilidad laboral } \\
\text { - Pago por tiempo de trabajo } \\
\text { - Escalafón rígido } \\
\text { - Concursos para plazas } \\
\text { - Incentivos normativos } \\
\text { - Racionalización de sistemas } \\
\text { administrativos } \\
\text { - Políticas de personal }\end{array}$ & $\begin{array}{l}\text { - Desregulación } \\
\text { - Flexibilización laboral } \\
\text { - Cambio en los mecanismos de pago } \\
\text { - Nuevos procesos de trabajo } \\
\text { - Protocolización } \\
\text { - Gestión de la calidad y la productividad } \\
\text { - Gestión descentralizada } \\
\text { - Políticas de desarrollo de } \\
\text { recursos humanos }\end{array}$ \\
\hline
\end{tabular}

desarrollistas y sus aparatos prestadores de servicios en la etapa de apogeo del estatismo, pero ello no significó la automática defunción del patrimonialismo. Mas bien éste encontró un nuevo espacio para recrearse, articulándose no sin conflicto el burocratismo y el patrimonialismo.

Encima de esta extraña mezcla hace una década llegó el gerencialismo -un tipo de gerencialismo, muy economicista, propio de las reformas de primera generación- intentando desburocratizar y desregular un campo insuficientemente burocratizado y regulado. Incluso en los tiempos de apogeo de las concepciones del Estado Benefactor, nunca llegamos a construir verdaderos sistemas de salud en nuestras latitudes, por lo menos en la mayoría de países andinos. Se puede entender, entonces, las dinámicas cruzadas que se han generado.

Por eso, nos parece, debemos tratar de superar la idea de que existe ya una nueva situación laboral y de gestión en los sistemas de salud andinos, ante la cual no se adecúan los recursos humanos. Esta argumentación es la conclusión lógica del esquema bipolar del que hablamos. En este discurso hay una sobrevaloración del nivel de implementación de las reformas sectoriales que no aplica a los países andinos, salvo Colombia. Este esquema de interpretación, en que los modelos institucionales están bien pero la gente que habita en ellos está mal, deriva de una lectura en blanco/ negro de los problemas de las reformas y en particular del campo de los recursos humanos en salud, una lectura acrítica de todo el paquete de reformas, como si fuera homogéneo, y una certidumbre en que fórmulas muchas veces y generales podrán tener absoluta viabilidad y factibilidad en culturas nacionales enteramente distintas, una omisión además de la diversidad de enfoques gerenciales hoy presentes en el mundo. Anotamos, de paso, que casi no hay algún análisis de los recursos humanos que no termine "inculpando a la víctima". Antes se les imputaba no entender la atención primaria o la salud pública y ahora no atender suficientemente la preocupación por los costos y la gerencia.

Los interventores e investigadores en el campo de recursos humanos ven, por eso, que se ha ori- 
ginado una situación paradójica en el campo de los recursos humanos en la última década, señalada por Brito: se quiere "cambiar radicalmente los servicios sin contemplar una inversión institucional, para acrecentar su máximo valor, los recursos humanos" $\left(^{1}\right)$. La nueva ideología del trabajo es la eficiencia, pero la nueva doctrina salarial y laboral no ha resuelto los desafíos de los incentivos y la motivación. En realidad, el nuevo programa de recursos humanos en la reforma necesita resolver algunos puntos anotados por la experiencia de la década de los 90, para adquirir claridad y dejar de ser contradictorio. Lo que denominamos "nueva gestión pública" en Latinoamérica cobija muchas cosas, desde el microfordismo en los procesos de trabajo hasta la aplicación indiscriminada o deforme de la teoría principal-agente, pasando por un constructivismo peculiar en los enfoques formativos.

De ahí que la década de los 90 deje la sensación de la falta de una direccionalidad clara capaz de ordenar la transición. Con variaciones menores, se mantienen casi todos los problemas clásicos de los recursos humanos en salud de hace quince años: baja disponibilidad, concentración urbana, formación biologista, enfoque pedagógico tradicional, régimen laboral desincentivador, niveles salariales bajos, escalafón "flexibilizado" por el clientelaje, baja productividad, alta conflictividad sindical y difícil regulación. No se puede decir que estos problemas hayan desaparecido. Algunos se han modificado, pero la mayoría persiste. La revisión hecha revela que los nuevos problemas son la multiplicación de las entidades educativas privadas, la superación de la baja disponibilidad -pero aún no de la concentración urbana de los profesionales de salud-, la saturación del mercado en ciertas áreas y la mayor visibilidad del desempleo laboral, la desregulación y desorden en la formación en salud, la superposición del biologismo clínico con el ascenso del gerencialismo en los postgrados, la implantación germinal de un nuevo régimen laboral flexible y la continuidad de la débil rectoría en el campo de los recursos humanos.

Vamos seguidamente a resumir brevemente algunos desafíos en el campo de recursos humanos en el área andina. Esta focalización de problemas se basa en una tipología de situaciones surgida del estudio de los recursos humanos en los países del Área Andina, reseñado aparte:

a) Colombia, caso de un país con una flexibilización laboral avanzada y en medio de un cambio sistémico;

b) Venezuela, Ecuador y Bolivia, casos de países en una situación de muy lenta asimilación de los cambios reformistas y fuerte resistencia gremial; y

c) Perú, caso de un país de generalización de un nuevo régimen laboral de contrataciones, difusión del gerencialismo, pero a la vez con una presencia gremial médica importante.

\section{De la baja disponibilidad a la sobreoferta urbana: el desafío de la desconcentración y des- centralización.}

Hasta no hace mucho, la visión demográfica de los recursos humanos limitaba el análisis a los problemas de disponibilidad cuantitativa. Hoy se viene trasladando el énfasis a la disponibilidad de capacidades (o competencias), porque en alguna medida se ha resuelto el problema clásico de la disponibilidad por 10000 habitantes en el área andina, por el incremento general de los recursos humanos en salud.

En realidad se ha pasado de la baja disponibilidad a la sobreoferta profesional, sobre todo en las zonas urbanas, precisamente por estar aún pendiente de solución el problema de la distribución de profesionales y técnicos en cada país. En esto, la descentralización en salud es una tendencia continental y mundial, que en el caso andino viene promoviendo un avance parcial en la redistribución del personal. El nuevo desafío ya no es tanto el número de profesionales sino su distribución y sus competencias.

Entre los años 1988 y 1997, el número de médicos se incrementó en 68,8 \% en el área andina, pasando de 91504 a 154 531. El mayor incremento se dio en Ecuador, donde la fuerza laboral en salud se duplicó durante el período, siguiéndole Venezuela, donde el incremento fue de $89,5 \%$, Colombia, con $46,5 \%$ y Perú con $36,6 \%$. En con- 
junto, la disponibilidad de médicos por 10000 habitantes entre los años 1992 y 1997 creció en 2,77 puntos, creciendo más en Bolivia $(8,48)$ y Venezuela $(7,47$ puntos) y menos en Colombia y Perú. Venezuela es el país andino con una mayor proporción de médicos por 10000 habitantes $(23,63)$; mientras que Perú es el que presenta la proporción más reducida $(11,20$ médicos por 10 000 habitantes) ${ }^{(2-4)}$ (Tablas 2 y 3 ).

Con relación al número de enfermeros, en el período 1988-1997 éste creció en 53,8\% en la subregión, algo menos que el crecimiento de los médicos. Llama la atención el gran incremento de esta categoría laboral en Bolivia, donde se triplicó; y en Ecuador, donde se duplicó. En Colombia y Perú el crecimiento fue más cercano al promedio ( $40 \%$ y $78 \%$, respectivamente), mientras que en Venezuela, el crecimiento en la disponibilidad de enfermeras fue muy reducido, apenas de $2 \%$. Sobre la disponibilidad de enfermeras por 10000 habitantes, en 1997 había 7,22 enfermeras por 10000 habitantes, 1,26 más que en 1992.

De otro lado, como conjunto, el número de odontólogos creció en $64,9 \%$ en la subregión

Tabla 2.- Médicos en la subregión andina, años 1988, 1992 y 1997.

\begin{tabular}{lrrrr}
\hline $\begin{array}{l}\text { Subregión } \\
\text { andina }\end{array}$ & 1988 & 1992 & 1997 & $\begin{array}{c}\text { Incremento } \\
1988-1997 \\
(\%)\end{array}$ \\
\hline Bolivia & 6819 & $3392 *$ & 10095 & 33,5 \\
Colombia & 29353 & 36551 & 43000 & 46,5 \\
Ecuador & 9901 & 15737 & 20243 & 104,5 \\
Perú & 20031 & 23771 & 27375 & 36,6 \\
Venezuela & 28400 & 32616 & 53818 & 89,5 \\
Total & 91504 & 112067 & 154531 & 68,8 \\
\hline
\end{tabular}

Fuente: Elaboración propia sobre la base de datos de la OPS (La Salud en las Américas, Edición 1990, 1994 y 1998) y a informes de países.

* Este dato procede del Ministerio de Previsión Social y Salud Pública de Bolivia. Organización Panamericana de la Salud. Representación en Bolivia. Cuadros Preliminares de Información sobre la Formación de Recursos Humanos en Bolivia en el Área de Salud, 1983-1992. La Paz, octubre de 1993.
Tabla 3.- Razón de médicos por 10000 habitantes, 1992 y 1997.

\begin{tabular}{lccc}
\hline $\begin{array}{c}\text { Subregión } \\
\text { andina }\end{array}$ & $\begin{array}{c}\text { Razón } \\
\text { por } 1000 \\
\text { habitantes } \\
1992\end{array}$ & $\begin{array}{c}\text { Razón } \\
\text { por } 1000 \\
\text { habitantes } \\
1997\end{array}$ & $\begin{array}{c}\text { Varia- } \\
\text { ción }\end{array}$ \\
\hline Bolivia & 4,51 & 12,99 & $+8,48$ \\
Colombia & 10,94 & 11,60 & $+0,66$ \\
Ecuador & 14,24 & 16,96 & $+2,72$ \\
Perú & 10,59 & 11,20 & $+0,61$ \\
Venezuela & 16,16 & 23,63 & $+7,47$ \\
Total & 11,65 & 14,42 & $+2,77$ \\
\hline
\end{tabular}

Fuente: OPS, La Salud en las Américas, 1998.

durante el período 1988-1997. El mayor crecimiento se dio en Ecuador; en Perú y Venezuela el crecimiento se dio en valores cercanos al promedio de la subregión (Tabla 4).

En general, en el área andina los profesionales de salud se incrementaron en forma importante en los últimos diez años. Esta situación se da por primera vez en la historia de los países andinos.

Lo que es antiguo es la distribución de los recursos humanos, concentrada en las áreas urbanas y en las zonas de mayor desarrollo relativo. Según la OPS, la relación entre las regiones con mayor concentración de médicos y la de menor presencia de los mismos es de 46,9 en Perú, 4,0 en Ecuador y de 1,9 en Bolivia $\left({ }^{5}\right)$.

\section{De los nombramientos a los contratos: el desafío irresuelto del nuevo régimen laboral.}

Todos los países del Área Andina están atravesados por el dilema irresuelto sobre el tipo de régimen laboral y de mecanismo de pago más aptos a la búsqueda de sistemas de atención más equitativos, sustentables, eficaces, eficientes y de calidad.

En general, como se ha anotado, en la mayoría de países andinos (Ecuador, Bolivia, Venezuela) todavía sigue primando el régimen de nombramientos, pero la tendencia es a la desestabilización del régimen laboral burocrático, que se basa en plazas o cargos, estabilidad laboral y el pago por tiempo de trabajo. Esto no viene significando necesa- 
Tabla 4.- Enfermeros en la subregión andina, años 1988, 1992 y 1997.

\begin{tabular}{lrrrr}
\hline $\begin{array}{l}\text { Subregión } \\
\text { andina }\end{array}$ & 1988 & 1992 & 1997 & $\begin{array}{c}\text { Incremento } \\
1988-1997 \\
(\%)\end{array}$ \\
\hline Bolivia & 2751 & -- & 5393 & 96,0 \\
Colombia & 11840 & 15251 & 16560 & 39,9 \\
Ecuador & 2771 & 5538 & 8362 & 201,8 \\
Perú & 15748 & 19612 & 28063 & 78,2 \\
Venezuela & 14389 & 15026 & 14676 & 2,0 \\
Total & 47499 & 55427 & 73054 & 53,8 \\
\hline
\end{tabular}

Fuente: Elaboración propia sobre la base de información de la OPS, La Salud en las Américas, ediciones 1990, 1994 y 1998, y a informes nacionales.

riamente el paso a regímenes laborales y de pago basados en sistemas de incentivos, como preveen los modelos de reforma. En los países en que más ha avanzado la flexibilización (Colombia y Perú), lo que viene cambiando es la forma de vinculación laboral (de los nombramientos a los contratos temporales), pero no se instala un sistema de pago basado en productividad, a ser cuantificado en servicios, casos resueltos o cualquier otro tipo de unidad de medida. Los contratos son con pago por tiempo de trabajo y su renovación queda a entera discreción del empleador. La construcción de sistemas de indicadores de productividad y de monitorización de la prestación, necesarios para un sistema de incentivos, viene siendo muy lenta, de tal forma que se ha generado una desestabilización de la institucionalidad laboral. El viejo régimen propiciaba la improductividad, pero el nuevo régimen temporal afecta la identidad institucional y el compromiso con la visión-objetivo, bases para un trabajo eficiente y dedicado.

Hay un interés masivo en la temática de los mecanismos de pago y en el debate sobre las bondades o defectos del pago "per capita", por "caso atendido" o por "grupos de diagnósticos relacionados", pero muy escasa operacionalización de los mismos $\left(^{6}\right)$. Incluso en Colombia, el país líder en la aplicación de los nuevos enfoques de reforma, Castellanos informa su introducción limitada aún ${ }^{7}$ ). En el Sistema de Seguridad Social en Salud de Colombia, explica Castellanos, la noción de capitación se ha utilizado como una forma de asignación de recursos por afiliado (Unidad de Pago por Capitación, UPC), sin derivar sus consecuencias posibles en términos de remuneración del trabajo profesional.

Se puede decir así, que en toda la subregión asistimos a un proceso de transición aún no acabado, de formas tradicionales de vinculación laboral y de pago del trabajo en salud hacia nuevas formas que aún no han cuajado del todo.

3. Del empobrecimiento de los salarios al doble régimen salarial usando los proyectos de cooperación: el desafío salarial y la reinvención de la carrera pública.

El empobrecimiento de los sueldos en el estado se ha hecho más pronunciado. En Venezuela, los odontólogos del sector público ganan $46 \%$ del sueldo de sus colegas en el sector privado y los sueldos promedio de los profesionales y técnicos del Ministerio de Salud cayeron entre 1988 y 1997 de 385 a 210 dólares $\left({ }^{8}\right)$. En Ecuador, los sueldos médicos no sobrepasan los 300 dólares mensuales y promueven la emigración profesional a Colombia y Chile $\left({ }^{9}\right)$. En el Perú, el Colegio Médico calcula los ingresos de la mayoría de médicos entre 400 y 800 dólares, sumando todas las horas extras y bonificaciones por trabajo en periferia, entre otros $\left({ }^{10}\right)$. En general los ingresos reales en salud del área andina están por debajo de los sueldos en Chile, Argentina o Brasil. Esto ha propiciado la emigración, el multiempleo y la fuga de personal calificado desde el sector público.

La respuesta ante esto no ha sido la recuperación salarial, sino el desdoblamiento del régimen salarial: salarios del estado para el personal y sueldos de la cooperación externa para el funcionariado. Se ha generado, desde los 90 en algunos países andinos, la contratación temporal de profesionales para la función directiva, como una forma de sortear los límites remunerativos vigentes en el sector público. Ello obviamente afecta la acumulación de capacidades, por tratarse de profesionales de paso.

A ello se añade, en el caso del Perú y Colombia, la desaparición del escalafón o carrera públi- 
ca, si bien éste es aún fuerte aún en Bolivia y Ecuador. El modelo burocrático afectaba la productividad, con el criterio de carreras basadas en realidad en el tiempo de servicio. Pero, la flexibilización con precarización del empleo -caracterización de la OIT del modelo que correspondió a la etapa del ajuste-, no sólo ha abierto espacio para la ampliación de los cargos de confianza hacia puestos no directivos y la expansión del clientelismo, sino que ha obviado la necesidad de establecer categorías profesionales y remunerativas que el personal podría desarrollar en las diferentes inserciones laborales a lo largo de su vida profesional. Sigue así pendiente el desafío de la reinvención de la carrera pública o servicio civil en salud, dentro de una política de recursos humanos basada en concursos públicos, en el reconocimiento a los niveles de experticia, la revisión de la política de remuneraciones actual para buscar capte cuadros calificados, la reorganización de las carreras y la promoción de la capacitación y desarrollo del funcionariado.

\section{Del arte clínico a la medicina gerenciada: entre los costos y la calidad de la atención.}

La actual reforma viene afectando la tradicional autonomía técnica de la profesión médica, esto es, su libertad en el campo de la clínica. Durante la etapa estatista nunca se tocó mayormente el campo del trabajo médico y se consintió en este terreno la prolongación de la regulación médica liberal. Esta autonomía técnica fue mayor en los países andinos, por el predominio del sector público, que en esas décadas se organizó sobre la base de las ideas-fuerza de cobertura y universalidad y no sobre la preocupación actual por el desempeño. Los catálogos de servicios, los protocolos de atención, los tarifarios, se desarrollaron más en los países donde hubo mayor presencia de los seguros privados.

En contraste, la actual reforma del sector salud viene siendo pro-regulacionista y no desrregulacionista, en este campo del trabajo asistencial. Las reformas tienden a flexibilizar la estabilidad laboral, pero no los procesos de trabajo, en donde implican más bien un "ajuste" sobre la profesión médica y todas las profesiones de salud en general.
La medicina gerenciada viene cerrando así el capítulo de la medicina liberal, pero mostrando a la vez la dificultad para la conciliación entre productividad y calidad. Durante toda la década última se ha anotado múltiples problemas derivados de la compulsión por el trabajo y los apremios por el cumplimiento de las cuotas laborales; el difícil equilibrio entre costos y calidad, cuando procedimientos diagnósticos o tratamientos contraponen a médicos y auditores o gerentes; así como el problema real de la deficiente o nula protocolización en los servicios. En realidad, apenas ha empezado en el área andina la definición técnica sobre los estándares de la atención.

\section{De la disminución del mercado laboral al in- cremento de la demanda educativa: el desafío de la formación de calidad y la acreditación.}

En toda el área andina, salvo Ecuador, hay en curso una multiplicación de la matrícula, de los egresados y de las instituciones educativas en salud, especialmente de las entidades privadas. En el Perú, en 1961 sólo existían cinco universidades con programas de formación en medicina, en 1980 ya eran 8 y a mediados de la década pasada, doce. Con la nueva Ley de Promoción de la Inversión en la Educación, dada en noviembre de 1996, llegaron a 24 en $1998\left({ }^{11}\right)$. En Bolivia, los matriculados y egresados crecieron entre 1983 y 1992 en $117 \%$ en odontología y $16,5 \%$ en medicina, triplicándose en los últimos seis años la oferta de cursos de pregrado en medicina y duplicado la de enfermería y odontología. Hacia 1992, el Ministerio de Previsión Social y Salud Pública intentó ordenar el campo de recursos humanos, formulando Lineamientos de Políticas al respecto, pero ello no detuvo la tendencia general al crecimiento desordenado de la oferta $\left({ }^{12}\right)$. En Colombia, la dación de la Ley 30 de la Educación Superior en 1993 promovió la multiplicación de instituciones y programas educativos, en alta proporción de naturaleza privada, quedando aminoradas en su poder las instancias reguladoras anteriores, la Asociación Colombiana de Facultades de Medicina (ASCOFAME) y el Consejo Nacional para el Desarrollo de los Recursos Humanos en Salud. En Venezuela, se informa de una saturación del mercado laboral por la "sobreproducción" de profesionales y una estabilización o ligero descenso de la matrícula, 
siendo el peso de los estudiantes y egresados de medicina (58\%) muy importante entre todas las demás profesiones y carreras en salud. Se puede apreciar que esta nueva problemática en el área andina en todas las profesiones y también a nivel de la formación de técnicos en salud.

Ello ha motivado movimientos de respuesta a esta no regulación de la educación privada en salud y consiguiente sobre-oferta educativa en salud. En Bolivia, el Ministerio de Educación emitió recientemente, el 22 de agosto de 2000, la Resolución Ministerial No 297/00, que aprobó la "Reglamentación para la apertura y funcionamiento de Programas o Carreras de Medicina en las Universidades Privadas" $\left({ }^{13}\right)$. En el Perú, el Colegio Médico consiguió se diese en 1998 la Ley $\mathrm{N}^{\mathrm{o}}$ 27154, que institucionalizó la Acreditación de las Facultades o Escuelas de Medicina, que por lo menos temporalmente bloqueó la creación de nuevas Facultades de Medicina, al estipularse estándares mínimos para ello. En Colombia, por el contrario, acaba de desaparecer la Dirección de Recursos Humanos en el Ministerio de Salud, integrándose sus funciones a otras áreas $\left({ }^{14}\right)$.

La puesta en marcha de la acreditación de Facultades o Escuelas de formación en salud es todavía muy incipiente en el área andina, pero es ya un compromiso ganado entre las mismas $\left({ }^{15,16}\right)$. En Bolivia se informa, sólo la Universidad de Cochabamba ha culminado ya su acreditación, realizándola en convenio con una universidad del exterior, siendo un proceso lento y muy dificultoso, por la presencia mayoritaria de las nuevas universidades privadas. En Colombia, el proceso de acreditación es voluntario y solamente se ha desarrollado de una manera muy incipiente en las universidades más consolidadas, que son las que probablemente ofrecen mejores programas. En Ecuador se informa la débil respuesta de las universidades a los nuevos desafíos y la no existencia aún de procesos de acreditación de instituciones de formación.

El hecho de que haya un incremento de la demanda educativa en salud sin un incremento equivalente en la demanda laboral se expresa en el mercado ocupacional. Hay cada vez más fuertes indicios de sobre-oferta y desempleo en salud, así como de emigraciones de profesionales. Ecuador informa de una corriente migratoria hacia la parte sur de Colombia y últimamente hacia Chile. Bolivia de la salida permanente de médicos hacia Brasil, Chile, Argentina, Perú y Estados Unidos. En Perú es notoria la emigración de médicos recién graduados hacia los Estados Unidos, España y Argentina. Hay así una relativa escisión de los dos mercados. El mercado educativo en salud es hoy más amplio y lucrativo que el laboral. Este tipo de desequilibrio tiende a hacerse estructural, con lo cual se podría afectar en los próximos años la calidad de la atención y procesar una reconfiguración de las profesiones de salud. La multiplicación de entidades formadoras podría tener como efecto la mediocrización de la formación en salud y un nuevo estatus de las profesiones de salud.

Sobre esto segundo, vale la pena recordar que la división del trabajo no abre automáticamente el espacio para el desarrollo de una profesión. El cuadro de profesiones de una sociedad es móvil y las fronteras de competencias y fueros se desplazan. Las categorías profesionales de una sociedad están en un proceso constante de profesionalización y desprofesionalización. Toda profesión necesita ser consciente, por tanto, de a dónde la conducen las tendencias sociales. La sociología de las profesiones enseña que las profesiones se constituyen cuando tienen un mercado de trabajo inviolable, una clientela fija y viven a tiempo completo de la actividad profesional $\left({ }^{17}\right)$. Esa es la diferencia entre una semiprofesión y una profesión. Esta última tiene un servicio específico considerado útil por la sociedad, servicio exclusivo de dichos profesionales. Por eso, éstos buscan el monopolio de dicha habilidad y la determinación del criterio de admisión. Cuando una profesión constituye su Colegio Profesional, persigue el control sobre el acceso, selección, proyección y reglamentación de la misma. Y si una profesión se dota de un código de ética, es para diferenciarse de los empíricos y legitimar a la profesión ante la sociedad.

Una sobreoferta podría repercutir regresivamente, determinando una involución del estatus de profesión a semiprofesión, como ha pasado en Latinoamérica con los maestros y viene sucediendo con los docentes universitarios de universidades públicas. El problema actual es que el principal factor de riesgo para la reconfiguración de las 
profesiones de salud no viene tanto de la reforma del sistema de salud, sino de los cambios en el sistema educativo. La multiplicación indiscriminada de Facultades de Medicina es, en términos reales, una reforma no sólo de la educación médica, sino del mercado laboral y la profesión misma.

\section{De la formación clásica universitaria a la educación permanente y continua: el desafío de la recertificación profesional.}

La revolución científico-médica en curso ha cambiado el enfoque anterior de la formación de recursos humanos dividida en dos etapas claramente separadas: la etapa universitaria y la etapa de capacitación o especialización periódica posterior. Las innovaciones en las ciencias de la salud son hoy tan veloces y profundas que los profesionales se ven impelidos cada vez más a la actualización sistemática. Se aprecia en la subregión andina, por eso, el ascenso visible de un movimiento de educación permanente y continua y un boom de la educación de posgrado.

La tendencia a la recertificación profesional viene siendo la forma institucional que asume este movimiento. Pero, el movimiento de educación permanente y continua es mucho más amplio que el de recertificación, que es inicial en la mayoría de países. En Bolivia, la existencia de un escalafón en el sector público promovió desde hace diez años la capacitación profesional y la acreditación de los cursos ante las autoridades para fines de ascensos, pero sólo ahora se propone organizar propiamente la recertificación $\left({ }^{18,19}\right)$. En Ecuador, la recertificación es incipiente y, en Colombia, cuenta con una fuerte oposición del gremio médico, porque se la interpreta como parte de la flexibilización laboral y la reducción del tamaño del estado. El Perú es el país andino más avanzado en cuanto a la recertificación médica, pero es un movimiento promovido desde el gremio médico y no cuenta aún con el reconocimiento del Ministerio de Salud. En el Perú se han dado 1,150 cursos en sólo dos años, 1999 y 2000, con 54,713 médicos participantes e incluso 11000 profesionales no médicos inscritos $\left({ }^{20}\right)$.

En realidad hay una diversidad de interpretaciones de la recertificación profesional, desde quie- nes la plantean como una evaluación para licenciamiento o habilitación periódica, quienes la asocian a los ascensos o carrera en el sector público, o quienes la conceptúan como un compromiso voluntario de actualización profesional. El arte para el desarrollo de la recertificación dependerá de si se plantea como amenaza o como oportunidad.

\section{De la formación tradicional a los nuevos en- foques educativos: la renovación de la educa- ción en salud.}

La "cuestión universitaria" sigue siendo un viejo problema en el campo de los recursos humanos en salud, en particular el problema de las universidades públicas. En algunos países andinos (Colombia), la universidad pública se ha modernizado, conservando su régimen autónomo. Pero en otros (Ecuador, Perú, en parte Bolivia), se ha venido dando en el sistema universitario público la expansión del alumnado y del cuerpo docente, asociado a una caída del gasto por estudiante y a bajas remuneraciones de los profesores $\left({ }^{21,22}\right)$. El reclutamiento y rendimiento del personal académico ha ido así hacia abajo. Como dice Romero, el profesorado universitario parece condenado a seguir la pérdida de estatus y de ingresos de los maestros de escuela y colegios; fenómeno, por lo menos, curioso en una sociedad que valora, así sea en el discurso, la ciencia y el conocimiento ${ }^{(23)}$. En el Perú se escogió en los 60 un sistema dual explícito, con universidades públicas abandonadas por el estado y universidades privadas de punta. Sólo hace 15 años, algunas universidades públicas reaccionaron y generaron una modernización universitaria. El problema generalizado en el área andina es, sin embargo, que la mayor parte de la formación de profesionales de salud proviene de universidades públicas, que el estado a veces maltrata financieramente, pero a cuyos egresados les exige las más altas competencias profesionales una vez incorporados a sus servicios.

En este contexto difícil, que incide en los estándares educativos, ha aparecido en los últimos años un nuevo movimiento de modernización educativa, concretado en innovaciones basadas en el enfoque constructivista y el enfoque de competencias, por ahora más visibles en la educación de 
posgrado que en el pregrado. Conforme ha resumido Granda $\left({ }^{24}\right)$, se viene modificando la formación clásica en salud en los países andinos de la siguiente manera:

a) Cambios en la educación en salud tendientes a basar la formación en problemas, asumir formas modulares, desarrollar competencias y evaluarse en función del desempeño.

b) Cambios en los temas de interés, ratificándose la primacía de las especializaciones asistenciales, motorizadas por las innovaciones científico-tecnológicas, pero cierto desplazamiento del interés de los propios profesionales asistenciales hacia el campo de la salud pública y la gerencia de servicios.

c) Cambios en la educación de posgrado, que viene incorporando la docencia semipresencial y a distancia, fortaleciendo la docencia descentralizada; pasando de la formación de posgrado centrada en maestrías hacia múltiples versiones novedosas (especialidades, diplomados, además de maestrías); y de una docencia centrada en el pensar a una docencia centrada en la acción, esto es, del saber a la competencia.

El proceso de implementación de estos nuevos enfoques educativos, así como de la acreditación de entidades formadoras, es todavía incipiente en la región, pero se desarrollará seguramente con más fuerza en los próximos años, superada la actual etapa introductoria.

\section{De la débil regulación a la desregulación: el desafío de la gobernabilidad del campo de los recursos humanos.}

La regulación en el campo de los recursos humanos siempre ha sido difícil, por los múltiples centros de iniciativa que intervienen en él (entidades formadoras, entidades proveedoras de servicios, estado, organizaciones profesionales, etc.). La tendencia en muchos países a la desregulación ha llevado esta situación a un nivel de fragmentación que resalta la necesidad de resolver la cuestión previa de la gobernabilidad en el campo de los recursos humanos en salud.

La mayoría de Direcciones de Recursos Humanos ministeriales y de instancias de concertación interinstitucional en educación en salud han sido afectadas en la década. En el Perú de los 90, la otrora poderosa Escuela Nacional de Salud Pública fue totalmente minimizada y sólo se mantiene formalmente la Dirección de Recursos Humanos en el Ministerio de Salud, no existiendo en la práctica una instancia de formulación ni de seguimiento de políticas de recursos humanos. Ahora último se le ha cambiado de nombre a la Escuela por el de Instituto de Desarrollo de Recursos Humanos, pero la verdad es que las cosas siguen igual. En Colombia, este año ha desaparecido la Dirección de Recursos Humanos y sus funciones han sido transferidas en forma dispersa a diferentes funcionarios de la Dirección de Desarrollo de Sistemas de Salud. El Instituto para el Fomento de la Educación Superior (ICFES), que regulaba la educación superior (estudios profesionales, tecnológicos y técnicos), el Consejo Nacional para el Desarrollo de los Recursos Humanos en Salud y la Asociación Colombiana de Facultades de Medicina (ASCOFAME), han sido desplazados en su poder real por la nueva Ley de Educación $\left({ }^{25-27}\right)$. En Venezuela, el organismo regulador en el campo de recursos humanos es la Dirección de Control Sanitario del Ministerio de Salud, que realiza el registro de títulos y el control de la práctica de los profesionales universitarios en ciencias de la salud, labores de hecho restringidas, que cumple limitadamente. En Bolivia, el Ministerio de Salud regula y supervisa la formación pública, pero corresponde al Ministerio de Educación hacer lo mismo con la formación privada, lo que constituye una dualidad y facilitó un descontrol de las universidades privadas que están en vías de superar con una nueva reglamentación.

Parte de este cuadro de creciente ingobernabilidad del campo de los recursos humanos en salud ha sido motivado por la introducción en algunos países de un tipo de gerencialismo no participativo en salud, que ha producido una incomunicación entre reformadores y reformados y un desacuerdo sobre la visión-objetivo a compartir. Se ha producido una reacción corporativa ante las reformas o modernizaciones en salud, muchas veces desde puntos de vista desfasados, pero otras desde la defensa legítima de los derechos ciudadanos de los profesionales de salud. Es probable, entonces, que la reconstrucción de la gobernabilidad 
en este campo suponga precisar el programa de recursos humanos en la reforma en salud.

\section{AGRADECIMIENTOS}

Mi agradecimiento al Dr. Pedro Brito, Coordinador del Programa de Recursos Humanos de la Organización Panamericana de la Salud, y a los Drs. Edmundo Granda, Gladys Zárate, María Cristina Taborda, Carmen Rosa Serrano y Lexin Arandia, responsables de las Áreas de Recursos Humanos de las representaciones de OPS en Ecuador, Perú, Colombia, Venezuela y Bolivia, respectivamente, por su valiosa y paciente colaboración con el autor de este informe.

El presente trabajo está basado en el capítulo final del informe de investigación sobre situación de los recursos humanos en Salud en el Área Andina, llevado adelante con el auspicio de la OPS y expuesto en la Reunión Internacional "El presente y el futuro en la formación, la práctica y la regulación del ejercicio profesional en ciencias de la salud", realizada en Guadalajara, México, entre el 22 y 25 de noviembre de 2000.

\section{BIBLIOGRAFÍA}

1. Brito P. Impacto de las reformas del sector de la salud sobre los recursos humanos y la gestión laboral. Revista Panamericana de Salud Pública 2000: 8(1-2): 43-54.

2. Organización Panamericana de la Salud. La Salud en las Américas, ediciones 1990, 1994 y 1998. Washington D.C: OPS, 1998. Informes de países.

3. Colombia. Ministerio de Salud. Censo del Recurso Humano y Dinámica Salarial, 1994-1998. Santafé de Bogotá, D.C., julio 1998.

4. Organización Panamericana de la Salud. Representación en Ecuador. Recursos Humanos en Salud en el Ecuador. Quito: OPS, septiembre 1993.

5. Organización Panamericana de la Salud. La Salud en las Américas, ibidem.

6. Gutiérrez C, y col. Las Formas de Contratación entre Prestadoras y Administradoras de Salud, sus Perspectivas en el Nuevo Marco de la Seguridad Social. Santafé de Bogotá, D.C.: Fundación Corona, Fundación Antonio Restrepo Barco, FES, 1995.

7 Castellanos J. Situación laboral del sector salud. Santafé de Bogotá, OPS, 1999.

8. Yejo N. Análisis de la situación de los planes y programas de capacitación y formación en salud. Caracas, Plan Rector de Formación de Recursos Humanos en Salud y Diseño de un Sistema de Planificación, Seguimiento y Evaluación de Planes y Programas definidos en esta Área, Producto 1, setiembre de 1999.
9. Granda E. Entrevista sobre la situación de los Recursos Humanos en salud en Ecuador. Lima, 08 de noviembre del 2000.

10. Castro J. Entrevista sobre los Recursos Humanos en salud en el Perú. Octubre 2000, Lima.

11. Castro J. Informe del Decano del Colegio Médico del Perú al III Seminario Nacional y Ier. Encuentro Latinoamericano de Certificación, Recertificación y Educación Médica Contínua. Lima, 9-11 noviembre del 2000.

12. Ministerio de Previsión Social y Salud Pública. Oficina Sectorial de Planificación y Desarrollo. Departamento Nacional de Recursos Humanos. Propuesta de Lineamientos de Políticas de Formación de Recursos Humanos en Salud. Documento de discusión. La Paz, Bolivia. 6 de marzo de 1992.

13. República de Bolivia. Reglamentación para la Apertura y Funcionamiento de Programas o carreras de Medicina en las Universidades Privadas. La Paz: Ministerio de Educación, Cultura y Deportes, Ministerio de Salud Pública y Previsión Social, Colegio Médico de Bolivia, Asociación Boliviana de Facultades de Medicina, año 2000.

14. Taborda C. Informe sobre la Situación de los Recursos Humanos en salud en Colombia. Bogotá, octubre del 2000.

15. Asociación Latinoamericana de Facultades y Escuelas de Medicina - ALAFEM. Memoria de la XVII Conferencia de ALAFEM. La Paz, 12-14 agosto de 1998.

16. Asociación Venezolana de Facultades y Escuelas de Medicina. Gestión de la calidad en la educación médica. Construcción de viabilidad. Caracas, 1994.

17. Machado M. Profissoes de saúde. Una abordagem sociológica. Rio de Janeiro, Editora Fiocruz, 1995, pp. 13-32.

18. Villagra O. La experiencia boliviana en Educación Médica Continua y Recertificación Profesional. Ponencia en el III Seminario Nacional y I Encuentro Latinoamericano de Educación Médica Continua, Certificación y Recertificación. Lima, 9-10 de noviembre del 2000.

19. Serrano C, Centeno R, Nassif M, Linger C. Reforma del Sector Salud: el Caso Boliviano. Educación permanente de personal de salud, pp. 91-94.

20. Castro. Informe, 2000, ibidem.

21. Rodríguez G. Las universidades privadas en Bolivia, 19661996. Informe de investigación. La Paz, 1996.

22. Rodríguez G. La vieja lógica de la relación Estado-Universidad. En: Desafíos de la Educación Superior, La Paz, pp. 87-90.

23. Romero S. Estado-Universidad-Sociedad: la necesidad de nuevas relaciones. En: Desafíos de la Educación Superior, s/f, La Paz, pp. 91-6.

24. Granda E. Entrevista, 2000, ibidem.

25. Taborda C. Informe 2000, ibidem.

26. Colombia. Ministerio de Salud. Decreto 1849 de 1992. Creación del Consejo Nacional para la Formación del Recurso Humano en Salud.

27. Colombia. Presidencia de la República. Decreto 1152 del 29 de junio de 1999 por el cual se reestructura el Ministerio de Salud como organismo de Dirección del Sistema General de Seguridad Social en Salud. Santafé de Bogotá, D.C., 1999. 